NT@UW-08-12

\title{
The neutron negative central charge density: an inclusive-exclusive connection
}

\author{
Gerald A. Miller \\ Department of Physics, University of Washington \\ Seattle, Washington 98195-1560 \\ John Arrington \\ Physics Division, Argonne National Laboratory \\ Argonne, Illinois 60439
}

\begin{abstract}
Models of generalized parton distributions at zero skewness are used to relate the behavior of deep inelastic scattering quark distributions, evaluated at high $x$, to the transverse charge density evaluated at small distances. We obtain an interpretation of the recently obtained negative central charge density of the neutron. The $d$ quarks dominate the neutron structure function for large values of Bjorken $x$, where the large momentum of the struck quark has a significant impact on determining the center of momentum, and thus the "center" of the nucleon in the transverse position plane.
\end{abstract}

PACS numbers: 13.40.Gp, 13.60.-r,13.60.Hb, 14.20.Dh

Keywords: form factors, charge densities, deep inelastic scattering

Much experimental technique, effort and ingenuity has been used recently to measure the electromagnetic form factors of the nucleon [1, 2, 3, 4]. These quantities are probability amplitudes that the nucleon can absorb a given amount of momentum and remain in the ground state, and therefore should determine the nucleon charge and magnetization densities.

In the non-relativistic case, the form factors are simply the Fourier transforms of the rest frame spatial distributions, and the charge and magnetization mean square radii are derived from the slope of the form factors at $Q^{2}=0$. In the relativistic case, this interpretation is not correct because the wave functions of the initial and final nucleons have different momenta and therefore differ, invalidating a probability or density interpretation. This is addressed by working in the Breit frame, where the magnitude of the initial and final nucleon momenta are identical. However, one needs boost corrections of order $Q^{2} / m^{2}$, where $m^{2}$ is the mass of the constituent particles to which the boost is applied, to relate the rest frame and moving nucleon wave functions. These corrections are model-dependent [5], so the use of the Breit frame does not provide a precise, model independent measure of the spatial distribution of the nucleon.

A recent work showed that it is possible to obtain a model-independent nucleon charge density [6]. In the infinite momentum frame (IMF), the two-dimensional Fourier transform of the elastic form factor $F_{1}$, provides a model-independent transverse charge distribution, $\rho_{\perp}(b)$, where $b$ is the distance from the center of momentum in the transverse plane. The use of existing data and convenient parameterizations $[7,8]$ yielded a central charge density of the neutron, $\rho_{\perp}^{n}(b=0)$, that is negative. We also note that the two-dimensional Fourier transform of $F_{2}$ can be interpreted as a magnetization density [9], and that this yields a difference between the magnetic and electric radii in the proton.
These findings appear to contradict previous understanding of the nucleon charge and magnetization distributions based on the model-dependent extraction of the rest frame charge distributions. The negative core of the neutron transverse density also contradicts previous intuition that the component in which the neutron is represented as a proton surrounded by a negatively charged pion cloud causes the central charge density to be positive. This negative core is a feature even in models that include a pion cloud effect to reproduce the measured values of $F_{1}^{n}$. It is therefore important to understand the differences between this model-independent transverse charge density and the rest frame charge density to fully understand the new features of the transverse spatial distributions.

Our goal is to obtain further information about the neutron charge density by using generalized parton distributions (GPDs) which contain information about the longitudinal momentum fraction $x$ as well as the transverse position $b$. Experimental information regarding the $x$ dependence is obtained by using GPDs to reproduce both deep inelastic scattering and elastic scattering data. Thus we use this inclusive-exclusive connection to better understand the central neutron charge density.

To start the analysis, we recall that form factors are matrix elements of the electromagnetic current operator $J^{\mu}\left(x^{\nu}\right)$ in units of the proton charge. The momentum transfer $q$ is space-like, so that $Q^{2} \equiv-q^{2}>0$. The normalization is such that $F_{1}(0)$ is the nucleon charge, and $F_{2}(0)$ is the proton anomalous magnetic moment. The Sachs electric and magnetic form factors are given by $G_{E}=F_{1}-\left(Q^{2} / 4 M^{2}\right) F_{2}$ and $G_{M}=F_{1}+F_{2}$.

The widely studied GPDs [10, 11] are of high current interest because they can be related to the total angular momentum carried by quarks in the nucleon. We consider the specific case in which the longitudinal momentum transfer $\xi$ is zero, and the initial and final nucleon 
helicities are identical $\left(\lambda^{\prime}=\lambda\right)$. Then, in the light-cone gauge, $A^{+}=0$, the matrix element defining the GPD $H_{q}$ for a quark of flavor $q$ and zero skewness is

$$
H_{q}(x, t)=\int \frac{d x^{-}}{4 \pi}\left\langle p^{+}, \mathbf{p}^{\prime}, \lambda\left|\widehat{O}_{q}(x, \mathbf{0})\right| p^{+}, \mathbf{p}, \lambda\right\rangle e^{i x p^{+} x^{-}},(1)
$$

where

$\widehat{O}_{q}(x, \mathbf{b}) \equiv \int \frac{d x^{-}}{4 \pi} q_{+}^{\dagger}\left(-\frac{x^{-}}{2}, \mathbf{b}\right) q_{+}\left(\frac{x^{-}}{2}, \mathbf{b}\right) e^{i x p^{+} x^{-}}$

We abbreviate $H_{q}(x, \xi=0, t) \equiv H_{q}(x, t)$ and $-t=-\left(p^{\prime}-\right.$ $p)^{2}=\left(\mathbf{p}^{\prime}-\mathbf{p}\right)^{2}=Q^{2}$. The simple form of $t$ results from its invariance under transverse boosts [12]: Lorentz transformations, defined by a transverse vector $\mathbf{v}$ that transform a four-vector $k$ according to $k^{+} \rightarrow k^{+}, \mathbf{k} \rightarrow \mathbf{k}-k^{+} \mathbf{v}$ and $k^{-}$such that $k^{2}$ is unchanged. These quantities are part of a kinematic subgroup of the Poincaré group that obey the same commutation relations as those among the generators of the Galilean transformations for nonrelativistic quantum mechanics in the transverse plane.

GPDs allow for a unified description of a number of hadronic properties [10]. The most relevant for us are that for $t=0$ they reduce to conventional PDFs, $H_{q}(x, 0)=q(x)$, and that the integration of the chargeweighted $H_{q}$ over $x$ yields the nucleon electromagnetic form factor:

$$
F_{1}(t)=\sum_{q} e_{q} \int d x H_{q}(x, t)
$$

The spatial structure of a nucleon can be examined [13, 14, 15, 16] using nucleonic states that are transversely localized. The state with transverse center of mass $\mathbf{R}$ set to $0,\left|p^{+}, \mathbf{R}=\mathbf{0}, \lambda\right\rangle$ is formed by taking a linear superposition of states of transverse momentum. Doing this requires the use of a frame with infinitely large $p^{+}$.

The impact parameter-dependent PDF 16 is the matrix element of the operator $\widehat{O}_{q}$ in the state $\left|p^{+}, \mathbf{R}=\mathbf{0}, \lambda\right\rangle$ :

$$
\rho_{\perp}^{q}(\mathbf{b}, x) \equiv\left\langle p^{+}, \mathbf{R}=\mathbf{0}, \lambda\left|\widehat{O}_{q}(x, \mathbf{b})\right| p^{+}, \mathbf{R}=\mathbf{0}, \lambda\right\rangle .
$$

We use the notation $\rho_{\perp}^{q}(\mathbf{b}, x)$ instead of the originally defined [16] $q(x, \mathbf{b})$ because the quantity truly is a density, giving the probability that the quark has a longitudinal momentum fraction $x$ and is at a transverse position b. The quantity $\rho_{\perp}^{q}(\mathbf{b}, x)$ is the two-dimensional Fourier transform of the GPD $H_{q}$ :

$$
\rho_{\perp}^{q}(\mathbf{b}, x)=\int \frac{d^{2} q}{(2 \pi)^{2}} e^{-i \mathbf{q} \cdot \mathbf{b}} H_{q}\left(x, t=-\mathbf{q}^{2}\right),
$$

with $H_{q}$ appearing because the initial and final helicities are each $\lambda$. A complete determination of $H_{q}(x, t)$ (with $t \leq 0)$ would determine $\rho_{\perp}(x, \mathbf{b})$.

One finds can extract the form factor $F_{1}$ [14 by integrating $\rho_{\perp}^{q}(\mathbf{b}, x)$ over all values of $x$, multiplying by the quark charge $e_{q}$, and summing over quark flavors $q$. The resulting IMF charge density in transverse space is

$$
\begin{aligned}
\rho_{\perp}^{N}(b) & \equiv \sum_{q} e_{q} \int d x \rho_{\perp}^{q}(\mathbf{b}, x) \\
& =\int \frac{d^{2} q}{(2 \pi)^{2}} F_{1}\left(Q^{2}=\mathbf{q}^{2}\right) e^{-i \mathbf{q} \cdot \mathbf{b}} .
\end{aligned}
$$

This quantity gives the charge density at a transverse position $\mathbf{b}$ irrespective of the longitudinal momentum fraction. The primary difference between the present charge density $\rho_{\perp}(b)$ and the older interpretation that the charge density as the three-dimensional Fourier transform of $G_{E}$ is that the present approach provides a model-independent, two-dimensional charge distribution in the plane transverse to the motion of the nucleon in the infinite momentum frame. The boost corrections here are simply kinematic and are incorporated in the formalism. In the older interpretation, the model-dependent boost corrections can not be avoided.

Our aim is to investigate $\rho_{\perp}(\mathbf{b}, x)$ to understand the origin of the neutron's negative central charge density. The quantities are not measured directly, but have been obtained from models that incorporate fits to parton distributions and electromagnetic nucleon form factors [17, 18, 19, 20]. This method exploits form factor sum rules at zero skewness, obtained neglecting the effects of strangeness, to obtain information regarding the valence quark GPDs, $H_{v}^{q} \equiv H^{q}-H^{\bar{q}}$. This yields the net contribution to the form factors from quarks and antiquarks, although it does not correspond to the valence distribution within a model for which sea distributions for quarks and antiquarks have different $x$ or $t$ dependences. To proceed further one must model the GPDs, and the results can be expected to depend on the chosen forms. Diehl et al. [17] use

$$
H_{v}^{q}(x, t)=q_{v}(x) \exp \left[f_{q}(x) t\right]
$$

where

$$
f_{q}(x)=\left[\alpha^{\prime} \log [1 / x]+B_{q}\right](1-x)^{3}+A_{q} x(1-x)^{2},
$$

is the form that gives the best fit to the data. The parameter $\alpha^{\prime}$ represents the slope of the Regge trajectory $\left(\alpha^{\prime}=0.9 \mathrm{GeV}^{2}\right)$, and the CTEQ6 PDFs [21] are taken as input. Here we use the best fit parameters, taken from the second line of Table 8 of [17]. These are $A_{u}=$ $1.26 \mathrm{GeV}^{-2}, B_{u}=0.59 \mathrm{GeV}^{-2}, A_{d}=3.82 \mathrm{GeV}^{-2}, B_{d}=$ $0.32 \mathrm{GeV}^{-2}$. We note that the labels $u$ and $d$ here refer to the $u$ and $d$ quarks in the proton. These correspond to $d$ and $u$ quarks in the neutron, if charge symmetry [22, 23, 24, 25] is upheld. It is well-known that for the proton, $2 d_{v} / u_{v}$ falls rapidly for large values of $x$, which means that $u$ quarks dominate the parton distribution for large values of $x$. This means that in the neutron, the $d$ quarks dominate the parton distribution for large values of $x$. The distributions of [18] have $A_{q}=B_{q}=0$ and $f_{q}(x)=\left[\alpha_{q}^{\prime} \log [1 / x]\right](1-x)$. Those of [19] have a 
more complicated form and also include the constraint that the nucleon consists of three quarks at an initial scale of $Q_{0}^{2}=0.094 \mathrm{GeV}^{2}$.

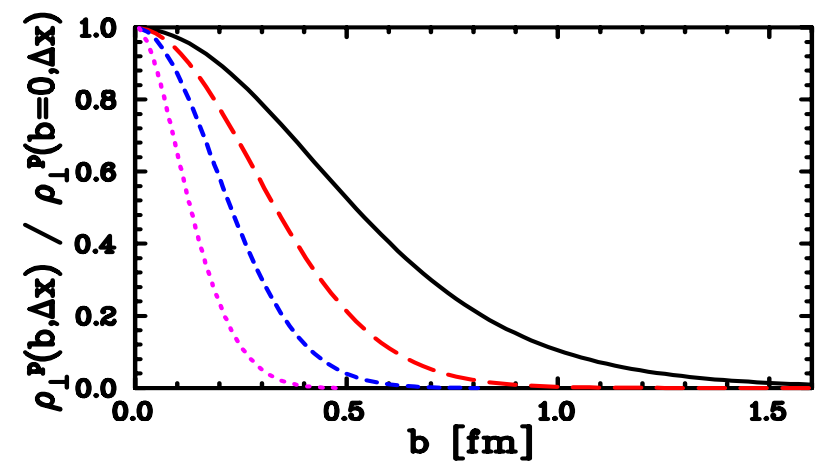

FIG. 1: (Color Online) The proton transverse charge density, $\rho_{\perp}^{p}(b, \Delta x)$, for quarks in different $\Delta x$ regions: $x<0.15$ (solid), $0.15<x<0.3$ (long-dash), $0.3<x<0.5$ (short-dash), and $x>0.5$ (dotted). The curves are calculated from the GPD of Ref. [17] and have been normalized to unity at $b=0$.

Our goal here is to examine the connection between regions of $x$ and regions of $b$. To do this we define

$$
\rho_{\perp}^{q}(b, \Delta x) \equiv \int_{\Delta x} d x e_{q} \rho_{\perp}^{q}(b, x),
$$

with $\rho_{\perp}^{p, n}$ being obtained from appropriate sums of $\rho_{\perp}^{q}$. This represents the contribution to the charge density from quarks in the $x$ region defined by $\Delta x$.

An important feature of the present approach is that these charge distributions are taken with respect to the center of momentum in the transverse plane. Thus, the transverse position $b$ is taken with respect to the momentum-weighted average position of all partons, including the struck quark. At low $x$, the struck quark has little impact on the center of momentum, and this corresponds to intuitive picture of spatial distribution. At large $x$, the struck quark plays a significant role in defining the $\mathrm{CM}$, and so distribution becomes localized at small values of $b$. This can be seen in Fig. 1, where for $x \approx 0.1$, the half-maximum width is $0.5 \mathrm{fm}$, while for $x \approx 0.8$, it is $0.12 \mathrm{fm}$. The curves have been scaled to yield unity at $b=0$, to emphasize the variation in width. The four $\Delta x$ regions yield $58 \%, 25 \%, 14 \%$, and $3 \%$ of the total charge, with the largest contributions coming from the bins with the smallest values of $x$. Thus the large $x$ quarks, dominantly $u$ quarks in the proton, play an increasingly prominent role in the charge distribution at small values of $b$. The figure obtained using the Guidal et $a l$, parameterization for the GPDs is barely distinguishable from Fig. 1. The GPDs of [19] also have a strong tendency to be constrained to smaller and smaller values of $b$ as the value of $x$ increases. We evaluate the GPDs of all three models using the starting scale $Q_{0}^{2}$ of each model.
Now consider the charge distribution of the neutron. We expect that the $d$ quarks dominate at large $x$ and therefore become important at small values of $b$. Because the distribution of quarks at large $x$ will be highly localized near $b=0$, a negative peak can be formed if the large $x$ distribution is sufficiently dominated by down quarks, thus yielding a significant contribution of negative charge at large enough $x$. At very low $x$ values, the valence distribution for up quarks in the neutron is roughly half that of the down quarks, $d_{v}^{n}(x) / u_{v}^{n}(x) \approx 2$, and the net charge coming from $u$ and $d$ quarks will approximately cancel, although the distribution as a function of $b$ need not be zero everywhere. Above $x=0.5, d_{v}^{n}(x)$ is at least three times the size of $u_{v}^{n}(x)$, and increases with $x$. So for $x>0.5$ the net impact to the charge distribution will be negative, and will be peaked at smaller values of $b$. We show this explicitly in Fig. 22 where we separate the contributions to the neutron charge density from $u$ and $d$ quarks based on the GPD fit of Ref. [17]. The distributions of [18] and [19] yield somewhat different results, but they exhibit the same qualitative behavior.



FIG. 2: (Color Online) The $u$ and $d$ quark contributions to the neutron transverse charge density, $\rho_{\perp}^{u}(b, \Delta x)$ and $\rho_{\perp}^{d}(b, \Delta x)$. The curves correspond to the same $\Delta x$ regions as in Fig. 1] The largest contributions come from small $x$, where $u$ and $d$ quarks contribute roughly equal amounts of charge. As one goes to larger $x$ values, the charge is shifted to smaller values of $b$, while at the same time the contribution from the up quarks drops rapidly with respect to the down quarks, due to the rapid falloff of the neutron $u$ to $d$ quark ratio at large $x$.

The next step is to examine the total charge distribution of the neutron. Fig. 3 separates the contributions from low and high $x$ regions. For $x<0.23$ the charge distribution is positive for $b<1.5 \mathrm{fm}$ and slightly negative distribution at larger radii. For $x>0.23$, the contribution is largely negative, and highly localized below $0.5 \mathrm{fm}$. The negative region at the center of the neutron transverse charge distribution arises a natural consequence of the model-independent definition of the charge density. 
The low momentum partons have a larger spatial extent and reproduce the intuitive result of the pion cloud picture: a positive core with a small negative tail at large distances.

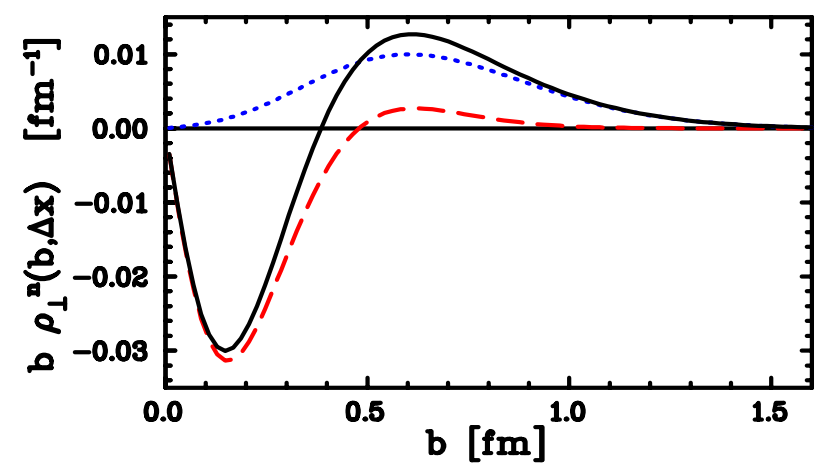

FIG. 3: (Color online) Transverse charge density for the neutron. The dotted line is the contribution from $x<0.23$, dashed is that for $x>0.23$, and the solid is the total.

A more intuitive picture of the charge distribution can be obtained by looking at the distribution of charge relative to the spectator partons, so that the struck quark does not influence the definition of the center of mass. This can be approximated by looking at the position of the struck quark relative to the spectators. We work in the transverse plane, with the origin set to the center of momentum, giving $\sum_{i} x_{i} \mathbf{b}_{\mathbf{i}}=0$. For a struck quark at $\left(x_{1}, \mathbf{b}_{\mathbf{1}}\right) \equiv(x, \mathbf{b})$, we can determine the momentumweighted spectator position, $\mathbf{b}_{\mathrm{spec}}$, and the relative distance from the struck quark to the spectator quarks:

$$
\begin{array}{r}
x_{1} \mathbf{b}_{1}+\sum_{i>1} x_{i} \mathbf{b}_{i}=x \mathbf{b}+(1-x) \mathbf{b}_{\mathrm{spec}}=0 \\
\mathbf{B}_{\mathrm{rel}}=\mathbf{b}-\mathbf{b}_{\mathrm{spec}}=\frac{\mathbf{b}}{(1-x)}=\mathbf{B}_{\mathrm{rel}} .
\end{array}
$$

We exhibit the dependence on $\mathbf{B}_{\text {rel }}$ by defining a function

$$
\rho_{\perp}^{\mathrm{Spec}}\left(\mathbf{B}_{r e l}, x\right) \equiv \rho_{\perp}\left(\mathbf{B}_{r e l}(1-x), x\right)
$$

which gives the probability that a struck quark of longitudinal momentum fraction $x$ is a distance $\mathbf{B}_{\text {rel }}$ away from the spectator center of momentum. Figure 4 shows this rescaled version of $\rho_{\perp}(b)$, with the contribution at each $x$ value normalized to unity at $b=0$. The quantity $\rho_{\perp}^{\mathrm{Spec}}\left(\mathbf{B}_{\mathrm{rel}}, x\right)$ can not be determined in a model independent manner, but may be a better approximation to our intuitive picture of the charge distribution, as it removes the influence of the struck quark on defining the center of the nucleon. While the charge distribution coming from very low $x$ quarks has a greater spatial extent, the decreasing width of the $\rho_{\perp}(b)$ distribution for large $x$ quarks is essentially completely removed when looking at $B_{\text {rel }}$.
Before concluding, it is worthwhile to comment on the relation between the present work and the difference between the electric and magnetic radii of the proton [9]. In the model-independent, IMF approach presented here, the electric and magnetic transverse radii have a clear connection to $F_{1}$ and $F_{2}$ and a Foldy [26] term causes a difference between the transverse radii. The Foldy term is responsible for most of the charge radius defined by $G_{E}$. Understanding the neutron's negative central density is more subtle and requires knowledge of $\rho(x, \mathbf{b})$.

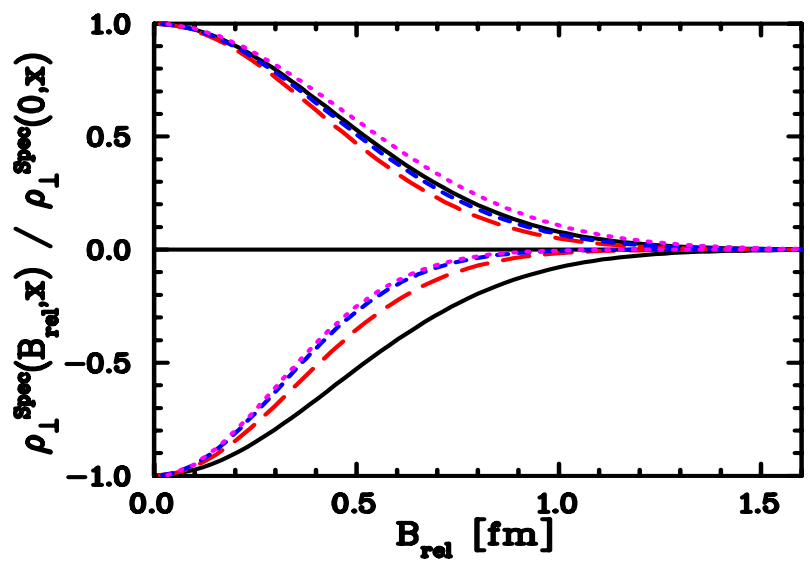

FIG. 4: (Color online) The $u$ and $d$ quark contributions to $\rho_{\perp}^{\mathrm{Spec}, n}\left(B_{\mathrm{rel}}, x\right)$ see Eq. (12). vs $B_{\text {rel }}$ for $x=0.1$ (solid), 0.3 (long-dash), 0.5 (short-dash), and 0.7 (dotted). The curves are scaled to unity at $B_{\text {rel }}=0$.

We summarize our findings with the statement that, using the model GPDs of Refs. [17, 18, 19], the dominance of the neutron's $d$ quarks at high values of $x$ leads to a negative contribution to the charge density which, due to the definition of $\mathbf{b}$, becomes localized near the center of mass of the neutron. This localization does not appear when examined as a function of the position of the struck quark relative to the spectators.

\section{Acknowledgments}

This work was supported by the U. S. Department of Energy, Office of Nuclear Physics, under contracts FG02-97ER41014 and DE-AC02-06CH11357. We thank D. Geesaman, P. Kroll and B. Wojtsekhowski for useful discussions. We thank the ECT* for hosting a workshop where many of the calculations we present were performed. 
[1] H. Gao, Int. J. Mod. Phys. E12, 1 (2003 [Erratum-ibid., $567,2003])$.

[2] C. E. Hyde-Wright and K. de Jager, Ann. Rev. Nucl. Part. Sci. 54, 217 (2004).

[3] C. F. Perdrisat, V. Punjabi, and M. Vanderhaeghen, Prog. Part. Nucl. Phys. 59, 694 (2007).

[4] J. Arrington, C. D. Roberts, and J. M. Zanotti, J. Phys. G34, S23 (2007).

[5] J. J. Kelly, Phys. Rev. C 66, 065203 (2002).

[6] G. A. Miller, Phys. Rev. Lett. 99, 112001 (2007).

[7] J. J. Kelly, Phys. Rev. C 70, 068202 (2004).

[8] R. Bradford, A. Bodek, H. Budd, and J. Arrington, Nucl. Phys. Proc. Suppl. 159, 127 (2006).

[9] G. A. Miller, E. Piasetzky, and G. Ron (2007), 0711.0972.

[10] X.-D. Ji, Phys. Rev. D55, 7114 (1997), hep-ph/9609381.

[11] A. V. Radyushkin, Phys. Rev. D56, 5524 (1997), hep$\mathrm{ph} / 9704207$.

[12] J. B. Kogut and D. E. Soper, Phys. Rev. D 1, 2901 (1970).

[13] M. Diehl, Eur. Phys. J. C25, 223 (2002).
[14] D. E. Soper, Phys. Rev. D 15, 1141 (1977).

[15] M. Burkardt, Int. J. Mod. Phys. A18, 173 (2003).

[16] M. Burkardt, Phys. Rev. D 62, 071503(R) (2000).

[17] M. Diehl, R. Feldmann, Th. an Jakob, and P. Kroll, Eur. Phys. J. C39, 1 (2005).

[18] M. Guidal, M. V. Polyakov, A. V. Radyushkin, and M. Vanderhaeghen, Phys. Rev. D 72, 054013 (2005).

[19] S. Ahmad et al., Phys. Rev. D 75, 094003 (2007).

[20] B. C. Tiburzi, W. Detmold, and G. A. Miller, Phys. Rev. D70, 093008 (2004), hep-ph/0408365.

[21] J. Pumplin et al., JHEP 07, 012 (2002).

[22] G. A. Miller, B. M. K. Nefkens, and I. Slaus, Physics Reports 194, 1 (1990).

[23] G. A. Miller, Phys. Rev. C 57, 1492 (1998).

[24] J. T. Londergan and A. W. Thomas, Prog. Part. Nucl. Phys. 41, 49 (1998), hep-ph/9806510.

[25] G. A. Miller, A. K. Opper, and E. J. Stephenson, Ann. Rev. Nucl. Part. Sci. 56, 253 (2006).

[26] L. L. Foldy, Phys. Rev. 83, 688 (1951). 